\title{
Antibacterial Activity of Amino Acid-Modified Cationic Dendrimers Loaded with a Mixture of Two Triterpenoid Acids ${ }^{+}$
}

\author{
Silvana Alfei ${ }^{1, *}$ and Anna Maria Schito ${ }^{2}$ \\ 1 Department of Pharmacy (DiFAR), University of Genoa, Viale Cembrano 4, I-16148, Genova, Italy \\ 2 Department of Surgical Sciences and Integrated Diagnostics (DISC), University of Genoa, Viale Benedetto \\ XV, 6, I-16132 Genova, Italy; amschitonige.it \\ * Correspondence: alfei@difar.unige.it; Tel.: +39-010-335-2296 \\ + Presented at the 2nd International Online-Conference on Nanomaterials, 15-30 November 2020; Available \\ online: https://iocn2020.sciforum.net/.
}

Published: 15 November 2020

\begin{abstract}
Searching for new devices capable to counteract the growing bacterial resistance, we reported the remarkable antimicrobial activity against several Gram-negative species of cationic dendrimers (CD), modified with lysine and histidine, also establishing that their cationic character, due to the lysine content, is essential for their potency. To develop new antibacterial agents, possibly active against Gram-positive species, cationic dendrimer complexes containing arginine and lysine, and trapping ursolic and oleanolic acids (UOACDs), two natural triterpenoids known for their activity against Gram-positive bacteria, were considered here as excellent candidates. Three selected UOACDs were tested unsuccessfully against Gram-negative strains, but successfully against 12 Gram-positive clinical isolates. The preliminary results obtained, in terms of MIC values, established that the antibacterial activity observed for UOACDs strongly depended on the number of cationic groups and the type of peripheral amino acids present in the CDs, and was attributable only to CDs and not to the presence and release of UOA. Lysine was critical for potency, while arginine redirected activity against Gram-positive species. A high cationic character, associated with the balanced content of lysine/arginine, produced a remarkable antimicrobial effect (MIC $=0.5-8.7 \mu \mathrm{M})$. More indepth investigations are underway to better characterize the activity of the developed argininecontaining CDs.
\end{abstract}

Keywords: new bactericidal agents; antibacterial polycationic dendrimers; arginine and lysine dendrimers; multi-drugs resistant Gram-positive bacteria; Enterococcus and Staphylococcus genera; ursolic and oleanolic acids.

\section{Introduction}

The rapid and worldwide increase in antimicrobial resistance among bacterial pathogens, frequently associated with therapeutic failures and high mortality rates, urgently requires alternative curative options, able to replace the no longer active antibiotics [1].

The most concern regards Gram-negative bacilli, including aerobic non-fermenting multi-drugs resistant (MDR) bacteria, such as Acinetobacter baumannii, Pseudomonas aeruginosa, and Stenotrophomonas maltophilia, which are emerging as clinically relevant superbugs, contributing significantly, with their worrying resistance levels, to numerous therapeutic failures [2]. However, antibiotic resistance has become also a major problem also in the treatment of infections caused by many Gram-positive bacteria. The most important Gram-positive resistant organisms include 
penicillin-resistant Streptococcus pneumoniae, methicillin-resistant Staphylococcus aureus (MRSA), S. epidermidis (MRSE) and Enterococcal species, such as Enterococcus faecium and E. fecalis that express high-level resistance to aminoglycosides and/or resistance to vancomycin (VRE) [3]. Frequently, these strains show multi-drug resistance, becoming tolerant to currently available agents, thus requiring innovative therapeutic strategies, including the administration of a higher antibiotic dosage, the use of alternative, non-conventional drugs, alone or in combination, and the development of new drugs. In this regard, an appealing class of potent unconventional antimicrobial molecules is represented by the natural cationic antimicrobial peptides (CAMPs) [4-7], characterized by a broad spectrum of action, being active on a wide variety of Gram-positive and Gram-negative bacteria, fungi, protozoa and yeast $[8,9]$.

According to several observations, CAMPs may damage and kill microorganisms by interfering with several specific and crucial bacterial vital processes [10], but mainly CAMPs are reported as membrane-active compounds and membranes disruptors [8-11].

Due to their cationic structures, due to the presence of the ammonium groups of some basic amino acids and of the $\mathrm{N}$-terminal groups of the fatty acids tails, CAMPs interact electrostatically with the anionic constituents of bacterial cell membranes, such as lipopolysaccharide (LPS) and phospholipids on the outer membrane (OM) of Gram-negative bacteria and with the teichoic acids on the surface of Gram-positive pathogens, thus managing to spread inside them.

These phenomena cause alterations in the integrity of the membranes, pore formation and permeabilization of the cell wall, that ultimately, lead to loss of bacterial cytoplasmic content and cell death [8-11].

Despite their efficacy, fast action, and a low incidence in the development of resistance [12], CAMPs are endowed with low biocompatibility and high toxicity against eukaryotic cells, which limit their clinical use [8].

In the last twenty years, less toxic, more stable and more low-cost synthetic mimics of CAMPs, including cationic peptides, positively charged polymers, and more recently polycationic dendrimers, containing different cationic structures, were developed $[8,13]$. Dendrimers represent a unique class of macromolecules, very different from linear polymers, extensively studied for applications in many sectors including the biomedical one [14-17]. Structurally different positively charged dendrimers, such as polyethyleneimine (PEI) dendrimers, organometallic dendrimers, ammonium-terminated, polypropylene imine (PPI), polyamidoamine (PAMAM) or peptide-based dendrimers, have been developed and successfully investigated as new unconventional devices to treat infections sustained by multi-drug resistant bacteria [13].

Among them, unmodified PAMAMs, such as the G7 PAMAM developed by Gholami et al. (2017) [18], proved to be very performant materials and to possess a strong broad-spectrum antibacterial activity in a number of case studies. Unfortunately, their clinical application is hampered by several issues, such as their low biodegradability, susceptibility to opsonization, high level of toxicity to mammalian cells, including hemolytic toxicity, cytotoxicity and fast clearance [14-17].

To address these issues, although they have still been little studied, polyester-based dendrimer scaffolds, peripherally functionalized with natural amino acids, have proven to represent very appealing alternative devices, endowed with considerable antibacterial activity and no cytotoxicity [19-21], also because of their good biodegradability [22-24].

In this regard, we recently published a study regarding the antibacterial effects of three fifth generation cationic polyester-based dendrimers (G5Ds) peripherally decorated with $L$-lysine (G5K), $L$-histidine $(\mathrm{G} 5 \mathrm{H})$ or a mixture $46 / 50$ of $L$-histidine and $L$-lysine (G5HK), selected among a library of 15 polycationic homo- and hetero-dendrimers, previously synthetized for gene therapy purposes [21,25].

Although considered non-active on E. coli, K. pneumoniae and all the Gram-positive strains, taken in consideration in the study, all G5Ds displayed remarkable activity against MDR non-fermenting Gram-negative species such as $P$. aeruginosa, S. maltophilia and A. baumannii. In particular, very low MIC values $(0.5-2.1 \mu \mathrm{M})$ were observed for the lysine-modified dendrimer G5K. G5K proved to be the most active dendrimer, even more active than the potent colistin (2.1 versus $3.19 \mu \mathrm{M})$ against $P$. 
aeruginosa, thus establishing the key role of the presence of cationic groups of lysine, to exert a selective antibacterial behavior towards these bacterial species [21]. On the contrary, the presence of histidine close to lysine, or the absence of lysine, tended to progressively decrease the antibacterial activity of the devices. Considering their lack of cytotoxicity and their activity comparable to, or even superior to that of antimicrobial dendrimers previously reported, G5Ds were the first example of polyester-based cationic dendrimers modified with single amino acids That were really effective in counteracting the alarming resistance of nosocomial of non-fermenting Gram-negative pathogens [21].

On this background of promising results, in this new study, it was explored the antibacterial activity of three new polyester-based amino acids-modified dendrimers, opportunely selected among a small library of six cationic dendrimers loaded with ursolic acid (UA) and oleanolic acid (OA) in mixture (UOA) [26] (Figure 1a). They had previously been prepared to make the two triterpenoids acids (UOA), otherwise not administrable in vivo because insoluble, water-soluble and administrable, and to enhance their properties [27], including antibacterial effects against Grampositive strains $[28,29]$.

a)

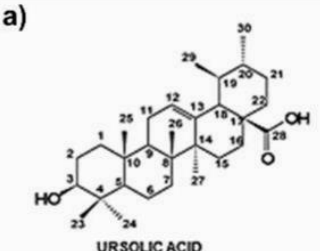

UOA

b)

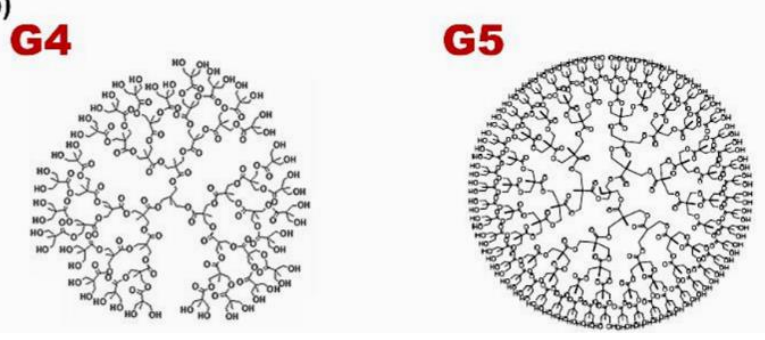

Figure 1. Structure of UA and OA, loaded as mixture into the cationic dendrimers (CDs) (a); (b) structure of the uncharged forth (G4) and fifth generation (G5) polyester-based inner scaffolds of the three CDs [27].

Structurally, the selected dendrimers possessed an inner uncharged polyester matrix of fourth (G4) or fifth (G5) generation (Figure 1b), peripherally esterified with mixtures having different content of arginine $(\mathrm{R})$ and lysine $(\mathrm{K})$ or with $\mathrm{R}$ alone. Additionally, they entrapped three, four or eight moles of UOA per mole of dendrimer. They were named G4R(16)K(19), G5R(38)K(30) and G5R(66), being the numbers in round brackets the content in amino acid residues of each dendrimer. Their stylized structures have been represented in Figure 2, which also reports the number of moles of UOA loaded respectively by one mole of each dendrimer. 


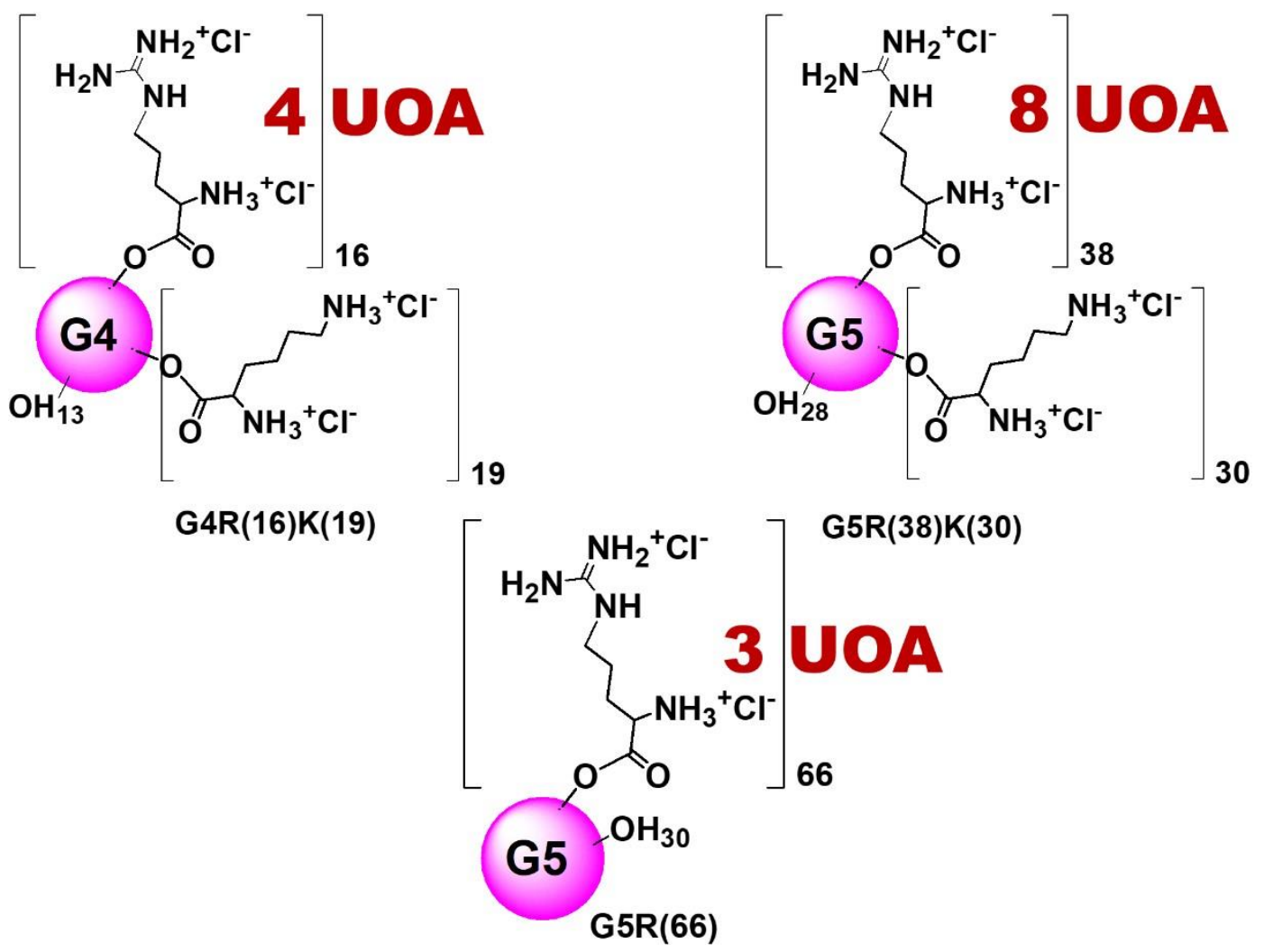

Figure 2. Schematic representations of the three GDs loaded with UOA [27].

As can be seen, all dendrimers retained a variable number of free hydroxyls during functionalization, as indicated near the $\mathrm{OH}$ groups in Figure 2.

\section{Materials and Methods}

\subsection{Microorganisms}

Two strains of Gram-negative bacteria and a total of 12 isolates belonging to 4 Gram-positive species were used in this study. All were clinical strains isolated from human specimens and identified by VITEK ${ }^{\circledR} 2$ (Biomerieux, Firenze, Italy) or Matrix-assisted laser desorption/ionization time-of-flight (MALDI-TOF) mass spectrometric technique (Biomerieux, Firenze, Italy). Of the tested organisms, 2 strains were of the Enterobacteriaceae family, (one E. coli and one K. pneumoniae resistant $(\mathrm{KCP})), 6$ strains belonged to the Staphilococcus genus, including 2 methicillin resistant S. auresus (MRSA) and one susceptible, 2 methicillin resistant S. epidermidis (MRSE) and one susceptible; 6 strains were of the Enterococcus genus (2 E. faecalis resistant to vancomycin (VRE) and one susceptible and 2 E. faecium VRE and one susceptible).

\subsection{Antimicrobial Assays}

The Minimal Inhibitory Concentrations (MICs) of the three CDs [G4R(16)K(19), G5R(38)K(30) and $G 5 R(66)]$ on the 14 pathogens were determined following the microdilution procedure detailed by the European Committee on Antimicrobial Susceptibility Testing EUCAST [30]. Briefly, overnight cultures of bacteria were diluted to yield a standardized inoculum of $1.5 \times 10^{8} \mathrm{CFU} / \mathrm{mL}$.

Aliquots of each suspension were added to 96-well microplates containing the same volumes of serial 2-fold dilutions (ranging from 1 to $1024 \mu \mathrm{g} / \mathrm{mL}$ ) of each dendrimer to yield a final concentration of about $5 \times 10^{5}$ cells $/ \mathrm{mL}$. The plates were then incubated at $37^{\circ} \mathrm{C}$. After $24 \mathrm{~h}$ of incubation at $37^{\circ} \mathrm{C}$, the lowest concentration of dendrimer that prevented a visible growth was recorded as the MIC. Concerning these early investigations, all MICs were obtained in duplicate, the degree of concordance was in all the experiments $2 / 2$ and standard deviation $( \pm \mathrm{SD})$ was zero. 


\section{Results and Discussion}

\subsection{Selection of Positively Charged Dendrimers GDs}

Confident that cationic polyester-based dendrimers, similar to the recently published G5Ds and active against Gram-negative species, could be endowed with similar antibacterial properties [21], we proceeded with the development of new cationic antibacterial dendrimers, with a more broad spectrum of action, or at least active against Gram-positive species on which previous G5Ds were ineffective.

In this regard, it was thought that the presence of moieties of compounds such as the triterpenoid acids UA and OA, previously shown to be active against species representing the genus Staphilococcus and Enterococcus $[28,29]$, could be helpful in achieving our goal.

Consequently, we thought to reconsider an available mini library of cationic dendrimers loaded with the UOA mixture, previously successfully synthetized to solubilize water-insoluble UA and OA extracted from Salvia corrugata [26], otherwise not bioavailable and not administrable in vivo [27].

After selecting the pull of the dendrimers loaded with UOA, a screening was carried out selecting high generation molecules (G4 and G5), since a higher generation would have allowed a high multivalence and therefore a higher content of amino acids and higher density of cationic charges $[9,18,21,31]$, essential for the interaction of the devices with bacterial membranes, for their destruction and killing of pathogenic microorganisms. Unlike the previous study [21], also a lower generation dendrimer, with significantly lower content in cationic groups was selected. This was done to explore whether a reduced cationic character could allow significant antibacterial activity to be maintained, and could help redirect activity even towards pathogens with less negatively charged external envelops, such as Gram-positive species. Since lysine was found to be essential for good antibacterial activity [21], two dendrimers containing different amounts of this amino acid were selected. However, to obtain antimicrobial devices active against various bacterial strains, the amino acid that accompanied lysine was modified.

Arginine was chosen instead of histidine, due to the presence of the guanidine group in its structure, as guanidinium homopolymers and copolymers were reported to be more effective against Gram-positive than against Gram-negative bacteria [8,9]. Finally, to evaluate the intrinsic efficiency of arginine, a fifth generation dendrimer containing exclusively arginine was chosen. Table 1 reports the main structural characteristic of the selected dendrimers and their physicochemical properties.

Table 1. Main structural characteristic and physicochemical properties of cationic dendrimers of this study [27].

\begin{tabular}{cccc}
\hline Features & G4R(16)K(19) & G5R(38)K(30) & G5R(66) \\
\hline Arginine, lysine, residual hydroxyls units & $16,19,13$ & $38,30,28$ & $66,0,30$ \\
UOA moles per dendrimer mole & 4 & 8 & 3 \\
UOA loading \% (wt/wt) & 12.6 & 12.7 & 5.0 \\
Cationic groups & 70 & 136 & 132 \\
Molecular Weight & 14600 & 29300 & 27400 \\
UOA released after 24 h (\%) & 75.2 & 65.9 & 65.2 \\
Z-potential (mV) & $24.8 \pm 0.2$ & $31.8 \pm 0.1$ & $34.0 \pm 0.6$ \\
Z-Ave size (nm) & $24.9 \pm 1.1$ & $20.3 \pm 3.1$ & $16.1 \pm 2.1$ \\
( $\mu$ g/10 mg) & 75.5 & 65.9 & 65.2 \\
\hline
\end{tabular}

\subsection{Antimicrobial activities of $G 4 R(16) K(19)$, G5R(38)K(30) and $G 5 R(66)$}

MIC values for all three dendrimers were obtained by analyzing a total of 14 strains of clinical origin. From preliminary results obtained on E. coli and K. pneumoniae, selected as representative of Gram-negative bacteria, it was established that CDs loaded with UOA were ineffective in inhibiting bacterial growth. For this reason, their activity has been studied more in deep against Gram-positive 
species, towards which significant antibacterial properties have been reported, especially against 12 strains, representatives of the genera Enterococcus and Staphylococcus (Table 2). In this regard, it was considered essential to establish whether the antibacterial activity exerted by the dendrimer complexes was due to a synergistic activity of the UOA with the cationic dendrimers, only to the cationic dendrimers or to the UOA released. Rational speculations have been possible, simply comparing the MIC values of the free UOA, the MIC values displayed by the UOACDs and the maximum concentration of UOA obtainable from these devices based on the observed MIC values (Table 2).

The maximum concentrations of UOA were calculated considering the UOA released after $24 \mathrm{~h}$ from $10 \mathrm{mg}$ of the different UOACDs [27]. In this regard, we are confident to correctly state hat the CD delivery systems themselves possessed antibacterial properties, that the presence of UOA did not contribute to the observed antibacterial potency of the dendrimer devices and that the inhibitory activity reported by the dendrimers was due solely to the cationic structures of dendrimer carriers themselves.

As confirmation to this assumption, the data obtained showed that, with the exception of one isolated case, the maximum concentration of UOA obtainable on the basis of the MIC values reported was far below the MIC values of free UOA, thus establishing that the antibacterial activity observed is attributable to the cationic dendrimer itself and not to the UOA.

In particular, G5R(66), containing only arginine, showed a similar antibacterial activity on Enterococcus and Staphilococcus, reporting comparable MIC values, and with a very narrow distribution, towards the two genera. Its activity against Staphilococci was lower than that of $\mathrm{G} 5 \mathrm{R}(38) \mathrm{K}(30)$, but higher than that of $\mathrm{G} 4 \mathrm{R}(16) \mathrm{K}(19)$, probably due to its lower? cationic character (70 cationic groups versus 132). On the contrary, against all Enterococci, G5R(66) showed much lower potency than the G4RK dendrimer (less cationic) and the G5RK dendrimer (with a similar cationic charge). From these findings, it can be concluded, that the genus Staphilococcus was more susceptible to the density of cationic groups present in the dendrimer structure, while the genus Streptococcus was more susceptible to the presence of lysine.

The greatest activity of the devices? was observed against the S. epidermidis 127 (MRSE) strain which was the most susceptible isolate of Staphilococcus to all UOACDs, reporting an order of potency in which $\mathrm{G} 5 \mathrm{R}(38) \mathrm{K}(30)>\mathrm{G} 5 \mathrm{R}(66)>\mathrm{G} 4 \mathrm{R}(16) \mathrm{K}(19)$ (Table 2). G4R(16)K(19) and G5R(38)K(30), containing both lysine and arginine, were much more active against the Enterococcus genus than on that of Staphilococcus, similarly to the free UOA mixture, displaying very low MIC values (2.2-4.4 $\mu \mathrm{M}$ and $0.5-1.1 \mu \mathrm{M}$ respectively). The most effective compound was G5R(38)K(30) which showed low MIC values also against the Staphilococcus genus $(2.2-8.7 \mu \mathrm{M})$.

It is noteworthy that the antibacterial activity of these cationic dendrimers was evident, on susceptible genera, regardless of the pattern of resistance to current antibiotics of the isolates evaluated.

In conclusion, it can be stated that the antimicrobial activity of the newly developed cationic materials depended mainly on the density of their cationic charge (and therefore on the number of cationic groups) and on the type of peripheral amino acids, (i.e., on the type of ammonium group responsible for the cationic character). From the MIC values reported in Table 2, it appears that, against the genus Staphylococcus, the antibacterial activity was proportional to the number of cationic groups, while against the genus Enterococcus a good antibacterial activity was due to the presence of the cationic groups of lysine. Indeed, G5R(66) not containing lysine, although possessing a number of cationic groups comparable to that of $\mathrm{G} 5 \mathrm{R}(38) \mathrm{K}(39)$, showed much lower antibacterial activity against all bacteria of genus Enterococcus (MIC values lower up to 37 times).

Moreover, G4R(16)K(19), despite possessing a far lower number of cationic groups than G5R(66), was significantly more active against all Enterococcus strains analyzed, probably due to the presence of lysine. Finally, since these first results showed that all dendrimer complexes were no effective against the Gram-negative strains considered, it can be established that the presence of the arginine guanidine group contributed to making the GCDs selective particularly for the Gram-positive strains [9]. 
Table 2. MIC values of the three dendrimers and of UOA, expressed as $\mu \mathrm{g} / \mathrm{L}$ and millimolarity (mM) against the Gram-positive strains tested in the study, and the max concentrations of UOA released according to the MIC values observed for each dendrimer complex according to its release profile previously reported [27].

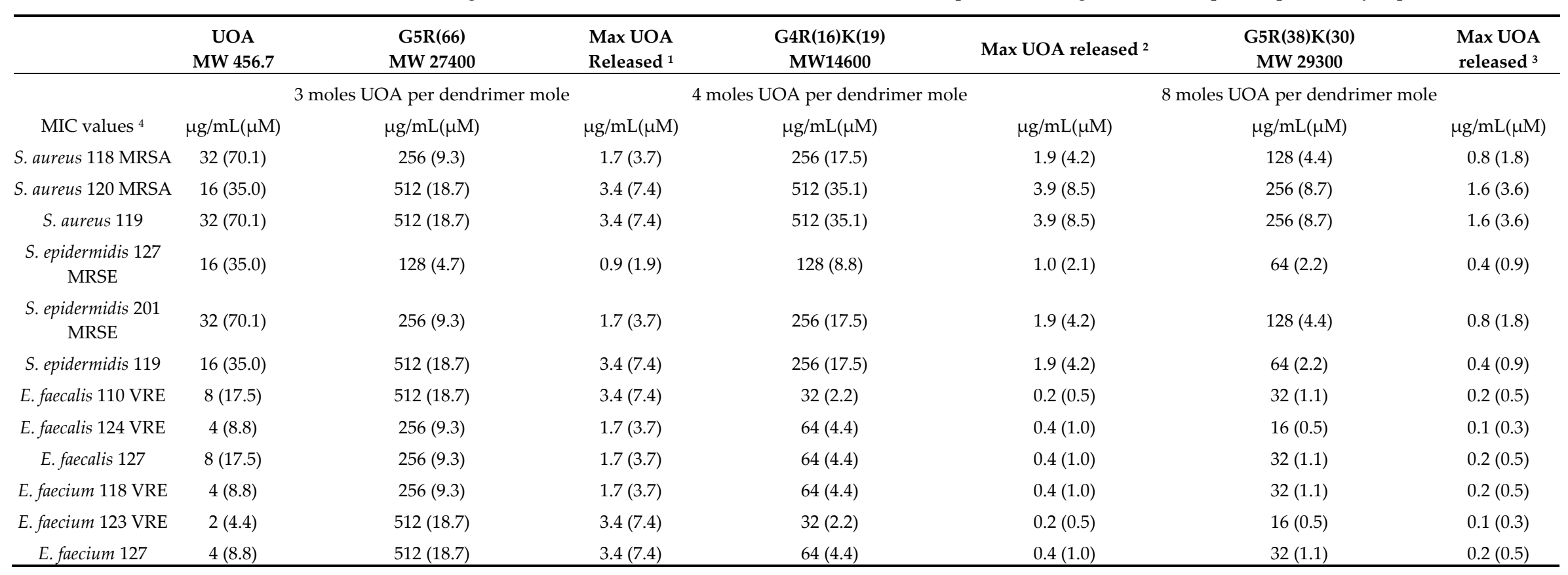

\footnotetext{
${ }^{1}$ by the MIC observed for G5R(66) after $24 \mathrm{~h} ;{ }^{2}$ by the MIC observed for G4R(16)K(19) after $24 \mathrm{~h} ;{ }^{3}$ by the MIC observed for G5R(38)K(30) after $24 \mathrm{~h} ;{ }^{4}$ experiments
} were made in duplicate and the concordance degree was $2 / 2$ and $\pm \mathrm{SD}$ was zero. 


\section{Conclusions}

The aim of the present work was to identify new and unconventional antimicrobial agents to replace traditional antibiotics, which are now less and less effective due to increasing bacterial resistance. In particular, we were mainly interested in antibacterial agents active against the main species of Gram-positive bacteria.

In a previous study carried out by our group (ref?), highly cationic dendrimers, modified with lysine and histidine, showed a selective potency for non-fermenting Gram-negative bacteria species, such as P. aeruginosa, A. baumannii and S. maltophilia. To pursue our objective, similar cationic materials were further selected, choosing them from a set of differently functionalized dendrimers, loaded with an extract of UO and OA (UOA), triterpenoid acids known for their antibacterial activity against Gram-positive species.

Although inactive against the Gram-negative bacteria tested, the selected dendrimers displayed considerable inhibiting properties against 12 Gram-positive bacterial strains of clinical origin, belonging to the genera Enterococcus and Staphylococcus.

All dendrimer devices exhibited an antibacterial activity attributable to their cationic structure and their amino acid content, and not to the presence of UOA, being the quantities of UOA produced as a function of the MIC values obtained, much lower than the MIC values observed for free UOA.

Interestingly, G5R(38)K(30) dendrimer, with the largest number of cationic groups and containing both lysine and arginine, was found to be the most active against all bacterial strains tested. In fact, it reported MIC values in the range of 2.2-8.7 $\mu \mathrm{M}$ against Staphylococcus strains, including MRSA and MRSE isolates, and in the range of 0.5-1.1 $\mu \mathrm{M}$ against Enterococcus strains, including VRE isolates.These findings confirmed that lysine is essential for a good antibacterial activity, and that the guanidinium group of arginine is capable of redirecting the activity of the dendrimers towards Gram-positive species.

Although more in-depth investigations are needed to better define the antibacterial activity of the arginine-containing dendrimers and to evaluate their cytotoxic profile, $\mathrm{G} 5 \mathrm{R}(38) \mathrm{K}(30)$, represents a biodegradable dendrimer device, which, harmonizing in its structure the correct ratio between the amino acids and an adequate number of cationic groups, is able to exert a strong antibacterial activity against Gram-positive species. The dendrimer $\mathrm{G} 5 \mathrm{R}(38) \mathrm{K}(30)$ is worthy of being considered a promising alternative to several commonly used antibiotics, now plagued by the burden of widespread resistance.

Author Contributions: The authors A.M.S. and S.A. contributed equally to the conceptualization, methodology, software, validation, formal analysis, investigation, resources, data curation, writing-original draft preparation, writing - review and editing and project administration for the present work. All authors have read and agreed to the published version of the manuscript.

Funding: This research received no external funding.

Acknowledgments: The authors thank Deirdre Kantz for language help and Sara Castellaro, which contributed to the synthesis of the polycationic dendrimers used in this study.

Conflicts of Interest: The authors declare no conflict of interest.

\section{References}

1. World Health Organization (WHO). Prioritization of Pathogens to Guide Discovery, Research and Development of New Antibiotics for Drug Resistant Bacterial Infections, Including Tuberculosis; WHO/EMP/IAU/2017.12; WHO: Geneva, $\quad$ Switzerland, Available online: https://www.who.int/medicines/areas/rational_use/PPLreport_2017_09_19.pdf?ua=1 (accessed on 15 October 2020).

2. World Health Organization (WHO). No Time to Wait: Securing the Future from Drug-Resistant Infections. Report to the Secretary-General of the United Nations. Interagency Coordination Group on Antimicrobial Resistance, WHO: Geneva, Switzerland, 2019. Available online: https:/www.who.int/antimicrobialresistance/interagencycoordination-group/IACG_final_report_EN.pdf?ua=1 (accessed on 15 October 2020). 
3. Koulenti, D.; Xu, E.; Mok, I.Y.S.; Song, A.; Karageorgopoulos, D.E.; Armaganidis, A.; Lipman, J.; Tsiodras, S. Novel Antibiotics for Multidrug-Resistant Gram-Positive Microorganisms. Microorganisms 2019, 7, 270 , doi:10.3390/microorganisms7080270.

4. Zasloff, M. Antimicrobial peptides of multicellular organisms. Nature 2002, 415, 389-395, doi:10.1038/415389a.

5. Seo, M.D.; Won, H.S.; Kim, J.H.; Mishig-Ochir, T.; Lee, B.J. Antimicrobial peptides for therapeutic applications: A review. Molecules 2012, 17, 12276-12286.

6. Gordon, Y.J.; Romanowski, E.G.; McDermott, A.M. A review of antimicrobial peptides and their therapeutic potential as anti-infective drugs. Curr. Eye Res. 2005, 30, 505-515.

7. Landman, D.; Georgescu, C.; Martin, D.A.; Quale, J. Polymyxins Revisited. Clinic. Microbiol. Rev. 2008, 21, 449-465.

8. Alfei, S.; Schito, A. Positively charged polymers as promising devices against multidrug resistant GramNegative bacteria: A Review. Polymers 2020, 12, 1195.

9. Kang, H.K.; Kim, C.; Seo, C.H.; Park, Y. The therapeutic applications of antimicrobial peptides (AMPs): A patent review. J. Microbiol. 2017, 55, 1-12.

10. Brogden, K.A. Antimicrobial peptides: Pore formers or metabolic inhibitors in bacteria? Nat. Rev. Microbiol. 2005, 3, 238-250.

11. Yang, L.; Gordon, V.D.; Trinkle, D.R.; Schmidt, N.W., Davis, M.A., DeVries, C., Som, A., Cronan, J.E., Tew, G.N.; Wong, G.C. Mechanism of a prototypical synthetic membrane-active antimicrobial: Efficient holepunching via interaction with negative intrinsic curvature lipids. Proc. Natl. Acad. Sci. USA 2008, 105, 20595-20600.

12. Spohn, R.; Daruka, L.; Lázár, V.; Martins, A.; Vidovics, F.; Grézal, G.; Méhi, O.; Kintses, B.; Számel, M.; Jangir, P.K.; et al. Integrated evolutionary analysis reveals antimicrobial peptides with limited resistance. Nat. Commun. 2019, 10, e4538.

13. Alfei, S.; Schito, A.M. From nanobiotechnology, positively charged biomimetic dendrimers as novel antibacterial agents: A review. Nanomaterials, 2020, 10, 2022, doi:10.3390/nano10102022.

14. Alfei, S.; Signorello, M.G.; Schito, A.; Catena, S.; Turrini, F. Reshaped as polyester-based nanoparticles, gallic acid inhibits platelet aggregation, reactive oxygen species production and multi-resistant Grampositive bacteria with an efficiency never obtained. Nanoscale Adv. 2019, 1, 4148-4157.

15. Alfei, S.; Catena, S.; Turrini, F. Biodegradable and biocompatible spherical dendrimer nanoparticles with a gallic acid shell and a double-acting strong antioxidant activity as potential device to fight diseases from “oxidative stress". Drug Deliv. Transl. Res. 2020, 10, 259-270.

16. Alfei, S.; Marengo, B.; Domenicotti, C. Polyester-based dendrimer nanoparticles combined with etoposide have an improved cytotoxic and pro-oxidant effect on human neuroblastoma cells. Antioxidants 2020, 9, e50.

17. Alfei, S.; Marengo, B.; Zuccari, G.; Turrini, F.; Domenicotti, C. Dendrimer nanodevices and gallic acid as novel strategies to fight chemoresistance in neuroblastoma cells. Nanomaterials 2020, 10, e1243.

18. Gholami, M.; Mohammadi, R.; Arzanlou, M.; Akbari Dourbash, F.; Kouhsari, E.; Majidi, G.; Mohseni, S.M.; Nazari, S. In vitro antibacterial activity of poly (amidoamine)-G7 dendrimer. BMC Infect. Dis. 2017, 17, 395, doi:10.1186/s12879-017-2513-7.

19. Stenström, P.; Hjorth, E.; Zhang, Y.; Andrén, O.C.J.; Guette-Marquet, S.; Schultzberg, M.; Malkoch, M. Synthesis and in Vitro Evaluation of Monodisperse Amino-Functional Polyester Dendrimers with Rapid Degradability and Antibacterial Properties. Biomacromolecules 2017, 18, 4323-4330, doi:10.1021/acs.biomac.7b01364.

20. Chen, A.; Karanastasis, A.; Casey, K.R.; Necelis, M.; Carone, B.R.; Caputo, G.A.; Palermo, E.F. Cationic Molecular Umbrellas as Antibacterial Agents with Remarkable Cell-Type Selectivity. ACS Appl. Mater. Interfaces 2020, 12, 21270-21282, doi:10.1021/acsami.9b19076. 343

21. Schito, A.M.; Alfei, S. Antibacterial activity of non-cytotoxic, amino acid-modified polycationic dendrimers against Pseudomonas aeruginosa and other non-fermenting Gram-negative bacteria. Polymers 2020, 12, 1818.

22. Alfei, S.; Castellaro, S. Synthesis and characterization of polyester-based dendrimers containing peripheral arginine or mixed amino acids as potential vectors for gene and drug delivery. Macromol. Res. 2017, 25, $1172-1186$ 
23. Alfei, S.; Catena, S. Synthesis and characterization of versatile amphiphilic dendrimers peripherally decorated with positive charged amino acids. Polym. Int. 2018, 67, 1572-1584, doi:10.1002/pi.5680.

24. Alfei, S.; Catena, S. Synthesis and characterization of fourth generation polyester-based dendrimers with cationic amino acids-modified crown as promising water soluble biomedical devices. Polym. Adv. Technol. 2018, 29, 2735-2749.

25. Alfei, S.; Castellaro, S.; Taptue, G.B. Synthesis and NMR characterization of dendrimers based on 2, 2-bis(hydroxymethyl)-propanoic acid (bis-HMPA) containing peripheral amino acid residues for gene transfection. Org. Commun. 2017, 10, 144-177.

26. Bisio, A.; Romussi, G.; Russo, E.; Cafaggi, S.; Schito, A.M.; Repetto, B.; De Tommasi, N. Antimicrobial activity of the ornamental species salvia corrugata, a potential new crop for extractive purposes. J. Agric. Food Chem. 2008, 56, 10468-10472.

27. Alfei, S.; Taptue, G.B.; Catena, S.; Bisio, A. Synthesis of Water-soluble, Polyester-based Dendrimer Prodrugs for Exploiting Therapeutic Properties of Two Triterpenoid Acids. Chin. J. Polym. Sci. 2018, 36, 999-1010, doi:10.1007/s10118-018-2124-9.

28. Wolska, K.I.; Grudniak, A.M.; Fiecek, B.; Kraczkiewicz-Dowjat, A.; Kurek, A. Antibacterial activity of oleanolic and ursolic acids and their derivatives. Cent. Eur. J. Biol. 2010, 5, 543-553, doi:10.2478/s11535-0100045-x.

29. Do Nascimento, P.G.; Lemos, T.L.; Bizerra, A.M.; Arriaga, Â.M.; Ferreira, D.A.; Santiago, G.M.; Braz-Filho, R.; Costa, J.G.M. Antibacterial and Antioxidant Activities of Ursolic Acid and Derivatives. Molecules 2014, 19, 1317-1327.

30. EUCAST. European Committee on Antimicrobial Susceptibility Testing. Available online: https://www.eucast.org/ast_of_bacteria/ (accessed on 15 October 2020).

31. Jain, A.; Duvvuri, L.S.; Farah, S.; Beyth, N.; Domb, A.J.; Khan, W. Antimicrobial polymers. Adv. Healthc. Mater. 2014, 3, 1969-1985, doi:10.1002/adhm.201400418.

Publisher's Note: MDPI stays neutral with regard to jurisdictional claims in published maps and institutional affiliations.

(C) 2020 by the authors. Submitted for possible open access publication under the terms and conditions of the Creative Commons Attribution (CC BY) license (http://creativecommons.org/licenses/by/4.0/). 